\title{
A COMPARISON OF SEVERAL COMMON MATHEMATICAL INDICES FOR MEASURING FUNCTIONAL DIVERSITY IN FOREST COMMUNITIES IN THE WULINGSHAN NATURE RESERVE, NORTH CHINA
}

\author{
SONG, N. Q. ${ }^{1}-$ ZHANG, Y. L. ${ }^{2}-$ ZHANG, J. T. ${ }^{2 *}$ \\ ${ }^{1}$ School of Chinese Materia Medica, Beijing University of Chinese Medicine, Beijing 102488, \\ China \\ ${ }^{2}$ Key Laboratory of Biodiversity Science and Ecological Engineering, Ministry of Education; \\ College of Life Sciences, Beijing Normal University, Beijing 100875, China \\ *Corresponding author \\ e-mail: Zhangjt@bnu.edu.cn; phone: +86-10-5880-3093; fax: +86-10-5880-7721
}

(Received 23 $3^{\text {rd }}$ Jan 2020; accepted 22 $2^{\text {nd }}$ May 2020)

\begin{abstract}
Functional diversity and its change mechanisms are important for forest conservation. Change patterns of functional diversity in forest communities along altitudinal and disturbance gradients in the Wulingshan Nature Reserve were analyzed using several common indices. Forty-one $20 \mathrm{~m} \times 20 \mathrm{~m}$ plots of forest communities were established along the altitudinal gradient. Plant species, functional traits and environmental variables were measured and recorded. Six functional diversity indices, Functional Attribute Diversity (FAD), Modified Functional Attribute Diversity (MFAD), Functional diversity based on dendrogram (FDp and FDc), Functional evenness (FEve) and Functional divergence (FDiv), were employed to quantify functional diversity. The results showed a great change in functional diversity of the forest communities in the Wulingshan Reserve. Functional diversity was significantly correlated with altitude and disturbance gradients. A non-linear "humped" change pattern along the altitudinal gradient and a non-linear or near linear decrease pattern along the disturbance intensity gradient were identified. Elevation and disturbance were the key factors influencing functional diversity. Functional diversity was closely related to species diversity in forests. The six common indices used were all effective and complementary to each other in functional diversity analysis in forests. MFAD, FEve and FDiv should be preferred in practice.
\end{abstract}

Keywords: environmental gradient, disturbance intensity, functional diversity index, functional traits, forest conservation

\section{Introduction}

Biodiversity and its conservation are two among the hottest topics in ecological studies (Austrheim, 2002). Species diversity, the measure of diversity in a community, is frequently studied in community ecology. Species diversity takes into consideration species richness and evenness, i.e. the total number of different species and the variation of abundance in individuals per species in a community (Perronne et al., 2014). However, the variation of species functions or in other words, functional diversity, has been poorly studied (Laliberte and Legendre, 2010). Functional diversity refers to the variation of species functions in a community (Petchey and Gaston, 2002; Zhang et al., 2015a). Plant species functions can be reflected by the type, change amplitude and stability of plant functional traits in a community (Villéger et al., 2008). Functional diversity is an important driver of ecological processes in a community (Zhang et al., 2014) and has become a key concept in ecology in recent years (Spasojevic et al., 2014; Rossi et al., 2020). 
The variation of functional diversity and its mechanism is the bases for forest conservation and have become attractive to forest ecologists and conservators (Muhumuza and Byarugaba, 2009; Pavoine and Bonsall, 2011). Nature reserves in the mountains are significant in forest conservation and ecological protection since most nature forests are distributed in mountainous regions with limited area in countries like China (Cui et al., 2008; Zhang et al., 2015a). Variation patterns of species diversity along altitudinal gradient have been studied frequently (e.g. Austrheim, 2002; Zhang et al., 2013; Perronne et al., 2014; Bricca et al., 2019) and most of them found a "humped" distribution pattern showing the maximum species diversity near the middle of the gradient (Austrheim, 2002; Otypkova et al., 2011; Zhang et al., 2014). However, functional diversity and its change patterns along environmental gradients have not been sufficiently studied (Albert et al., 2012; Zhang et al., 2015b).

The Wulingshan reserve is a famous eco-tourism destination for citizens in Beijing and Hebei Province (Xiang and Zhang, 2009). Forests are significant in the local economy and development and should be conserved effectively in this reserve. Some researches on eco-tourism resources and evaluation (Zhang et al., 2003), plant resources and floristic characteristics (He et al., 1992; Duan, 2009), the vertical distribution of forest communities (Liu and Xi, 1997), vegetation landscape patterns (Bai et al., 2006), and so on have been conducted in this Reserve. However, the variation of functional diversity associated with the major environmental gradients has not been examined. This study aimed to analyze the interdependencies of functional diversity, environmental variables and disturbance in forest communities, to compare the effectiveness of several common functional diversity indices, and to test the hypothesis that functional diversity peaks at an intermediate altitude and medium disturbance in forests in the Wulingshan Nature Reserve.

\section{Materials and methods}

\section{Study area}

The Wulingshan Nature Reserve, located at $117^{\circ} 17^{\prime}-117^{\circ} 35^{\prime} \mathrm{E}, 40^{\circ} 29^{\prime}-40^{\circ} 36^{\prime} \mathrm{N}$, is in the Miyun County of Beijing and Xinglong County in Hebei Province (Fig. 1). The reserve was established to protect the typical forest ecosystems and the northern limit of distribution of the rhesus macaque in 1984. It covers an area of 14,337 ha (Liu and Xi, 1997). Its highest peak, $2116 \mathrm{~m} \mathrm{ASL}$, is in the northern part of Xinglonh County. Its climate is deeply affected by the warm temperate southeastern monsoon. The annual mean temperature is $7.6^{\circ} \mathrm{C}$; the mean monthly temperature of July is $17.7^{\circ} \mathrm{C}$, whereas the mean monthly temperature of January is $-15.6^{\circ} \mathrm{C}$. The annual mean precipitation varies from $600 \mathrm{~mm}$ (northern slope) to $720 \mathrm{~mm}$ (southern slope). The main soil types include cinnamon soil in the lower area, brown forest soil in typical forest area and mountain meadow soil over the forest line of this mountain. The vegetation includes secondary forests with some plantations from 700 to $1900 \mathrm{~m}$ ASL and mountain meadows and scrublands above $1900 \mathrm{~m}$ ASL.

\section{Data collection}

Based on a general survey, 16 sampling points separated by $60-80 \mathrm{~m}$ in altitude were set up along the altitudinal gradient in forest area between 700 and $1900 \mathrm{~m} \mathrm{ASL}$ in the Wulingshan Reserve. Two to four plots $(20 \mathrm{~m} \times 20 \mathrm{~m})$ around each sampling point were 
established randomly. The cover, height, individual abundance and diameter at breast height $(\mathrm{DBH})$ for tree species and the height and cover for shrubs and herbs were measured and recorded for each plot. Tree height was measured using a height-meter (Haguang CGQ-1, 0-77 $\mathrm{m}$ ) and shrub and herb height using a tape ruler. DBH was measured using a caliper (Argus PiCUS Calliper, 0-150 cm). A total of 172 plant species were recorded in 41 plots.

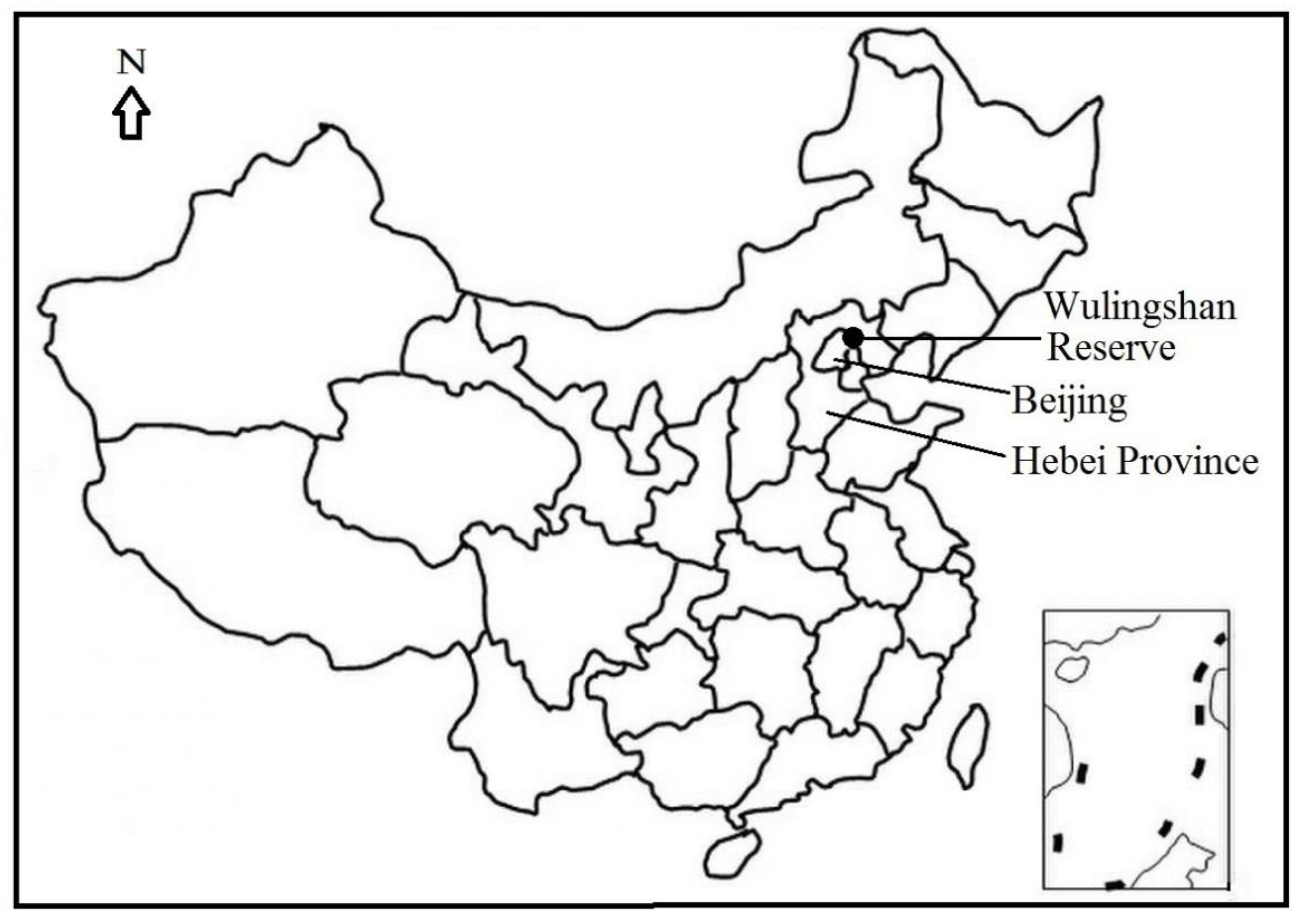

Figure 1. Geographical location of the study area, the Wulingshan Nature Reserve, China

Twelve plant functional traits were selected to reflect species functions in the plant community (Table 1). Photosynthesis pathway, pollination manner, seed dispersal, lifeform, root system and nitrogen-fixing type were identified from local flora. Leaf form, leaf hair, plant height, flowering date and period, and fruit maturity date were investigated in the field. Functional diversity indices were calculated using a data matrix of plant species by functional traits for each plot. There were 41 data matrices for 41 plots in total.

Five environmental variables, altitude, slope, aspect, soil depth and litter thickness, were measured and recorded for each plot. The elevation was measured using a GPS, the slope and aspect were measured using a compass meter, the soil depth was measured using a soil depth instrument and the litter thickness was measured using a ruler directly (Zhang et al., 2015a). The elevation, slope, litter thickness and soil depth were measured values, while the slope aspect was transformed to 1-8 values: $1\left(337.6^{\circ}\right.$ $\left.22.5^{\circ}\right), 2\left(22.6^{\circ}-67.5^{\circ}\right), 3\left(292.6^{\circ}-337.5^{\circ}\right), 4\left(67.6^{\circ}-112.5^{\circ}\right), 5\left(247.6^{\circ}-292.5^{\circ}\right), 6$ $\left(112.6^{\circ}-157.5^{\circ}\right), 7\left(202.6^{\circ}-247.5^{\circ}\right)$, and $8\left(157.6^{\circ}-202.5^{\circ}\right)$. The greater the value, the more sunlight is available.

The disturbance intensity from human activity (mainly tourism activity) was evaluated as scores of 1-5 based on tourist density, trampling extent on understory plants, distance from traveling pathway, number of pieces of garbage, and ratio of 
human accompanying plant species in a plot: 1 (non-obvious disturbance), 2 (weak disturbance), 3 (medium disturbance), 4 (heavy disturbance) and 5 (very heavy disturbance) (Zhang et al., 2013).

Table 1. Plant functional traits and their values in forest communities in the Wulingshan Nature Reserve, North China

\begin{tabular}{|c|c|c|}
\hline Functional trait type & Data type & Functional traits and values \\
\hline Photosynthesis pathway & Attribute value & $\begin{array}{c}1 \text { Crassulacean pathway, } 2 \text { C3 pathway, } 3 \text { between C3 } \\
\text { and C4 pathway, } 4 \text { C4 pathway }\end{array}$ \\
\hline Nitrogen-fixing & Attribute value & $\begin{array}{c}0 \text { No nitrogen-fixing, } 1 \text { Elaeagnaceae nitrogen-fixing, } 2 \\
\text { Leguminosae nitrogen-fixing, } 3 \text { mycorhiza nitrogen- } \\
\text { fixing }\end{array}$ \\
\hline Seed dispersal & Attribute value & $\begin{array}{l}1 \text { Automatic spreading, } 2 \text { gravity spreading, } 3 \text { wind } \\
\text { spreading, } 4 \text { animals spreading, } 5 \text { non-flowering plant }\end{array}$ \\
\hline Pollination method & Attribute value & 1 Anemophilous, 2 entomophilous, 3 self-pollinated \\
\hline Life-form & Attribute value & $\begin{array}{c}1 \text { Tree, } 2 \text { shrubs, } 3 \text { woody vine, } 4 \text { perennial herb, } 5 \\
\text { annual herb }\end{array}$ \\
\hline Leaf form & Attribute value & 1 Coniferous, 2 Broad leaf, 3 non leaf \\
\hline Leaf hairs & Attribute value & $\begin{array}{l}0 \text { No hair, } 1 \text { hairs on top surface, } 2 \text { hairs on back } \\
\text { surface, } 3 \text { hairs on both sides }\end{array}$ \\
\hline Root system & Duality value & 1 Taproot system, 2 Fibre system \\
\hline Plant height & Quantitative value & Measured value in $\mathrm{m}$ \\
\hline Flowering date & Quantitative value & Beginning month of flowering \\
\hline Flowering period & Quantitative value & Flowering months \\
\hline Fruit maturity date & Quantitative value & Beginning month of fruit mature \\
\hline
\end{tabular}

\section{Data analysis}

\section{Functional diversity indices}

Six common functional diversity indices were used to calculate functional diversity in forest communities:

\section{Functional attribute diversity (FAD)}

FAD is a measure of species dispersion in trait space and refers to the sum of the pairwise species functional distances (Walker et al., 1999):

$$
F A D=\sum_{i, j}^{S} d_{i j}
$$

where $d_{i j}$ is the functional distance between species $i$ and $j$ in functional trait space. $S$ is the number of species in a plot.

\section{Modified functional attribute diversity (MFAD)}

For MFAD, the functional unit was defined by combining the species with the same values in all the traits into one unit. The number of species will be reduced from $S$ to $M$ $(M \leq S)$ (Casanoves et al., 2011). 


$$
M F A D=\frac{\sum_{i, j}^{M} d_{i j}}{M}
$$

where $d_{i j}$ is the distance between functional units $i$ and $j$, and $M$ is the number of functional units.

Functional diversity based on dendrogram (FDp and FDc)

Functional diversity based on a dendrogram refers to the sum of branch length of the dendrogram produced by a clustering method based on the data matrix of $S$ species $\times N$ traits in a plot. $F D p$ is plot based functional diversity index and $F D c$ is community based index (Petchey and Gaston, 2006).

Functional divergence (FDiv)

Functional divergence is related to how abundance is distributed within the volume of functional traits space.

$$
F D i v=\frac{\sum_{i=1}^{S} w_{i}\left(d G_{i}-\overline{d G}\right)+\overline{d G}}{\sum_{i=1}^{S} w_{i}\left|d G_{i}-\overline{d G}\right|+\overline{d G}}
$$

$d G_{i}$ is the functional distance from species $i$ to the gravity center of species that form the vertices of the convex hull, and $\overline{d G}$ is the mean distance of the $S$ species to the gravity center, and $w_{i}$ is the relative abundance of species $i$.

Functional evenness (FEve)

FEve measures the regularity of spacing between species in the functional trait space and the evenness of the distribution of the species abundance. The minimum spanning tree (MST) was used to transform a multidimensional space to a distribution on a single axis. This index measures both the sum of branch lengths in the MST and the evenness of species abundance (Villéger et al., 2008). The index:

$$
F E v e=\frac{\sum_{b=1}^{S-1} \min \left(P E W_{b}, \frac{1}{S-1}\right)-\frac{1}{S-1}}{1-\frac{1}{S-1}}
$$

where: $P E W_{b}=\frac{E W_{b}}{\sum_{b=1}^{S-1} E W_{b}}, E W_{b}=\frac{d_{i j}}{w_{i}+w_{j}}$.

There are $S-1$ branches in the MST of $S$ species and each of the $b$ branch length is divided by the sum of the abundance of the species linked. $E W_{b}$ is the weighted evenness, $d_{i j}$ is the Euclidean distance between species $i$ and $j$, those involved in the 
branch $b$, and $w_{i}$ and $w_{j}$ are the relative abundance of these species. It is useful to compute the partial weighted evenness dividing by the sum of the across the branches.

Species diversity

Three species diversity indices, species number (as a richness index) $D=S$, Shannon-Wiener index $H^{\prime}=-\sum P_{i} \ln P_{i}$, Pielou evenness index $E=H^{\prime} / \ln (S)$, were used to measure species diversity in a community. Where $P_{i}$ is the relative abundance of species $i, P_{i}=N_{i} / N, N_{i}$ the abundance of species $i, N$ the sum of abundance for all species in a plot, $S$ the species number present in a plot.

Spearman correlation and regression were used to analyse the relationships of functional diversity, species diversity and environmental variables.

\section{Results}

Plant functional diversity demonstrated by the six indices showed great variations in forest communities in the Wulingshan Nature Reserve. FAD varied between 69.76 and 145.62 with a mean 107.99 and a standard deviation 18.10, MFAD between 3.81 and 5.92 with a mean 4.87 and a standard deviation 0.59, FDp between 6.44 and 9.97 with a mean 8.13 and a standard deviation 0.88 , FDc between 6.33 and 9.95 with a mean 8.31 and a standard deviation 0.90 , FEve between 0.68 and 0.88 with a mean 0.79 and a standard deviation 0.05 , and FDiv between 0.69 and 0.90 with a mean 0.81 and a standard deviation 0.05 (Fig. 2).
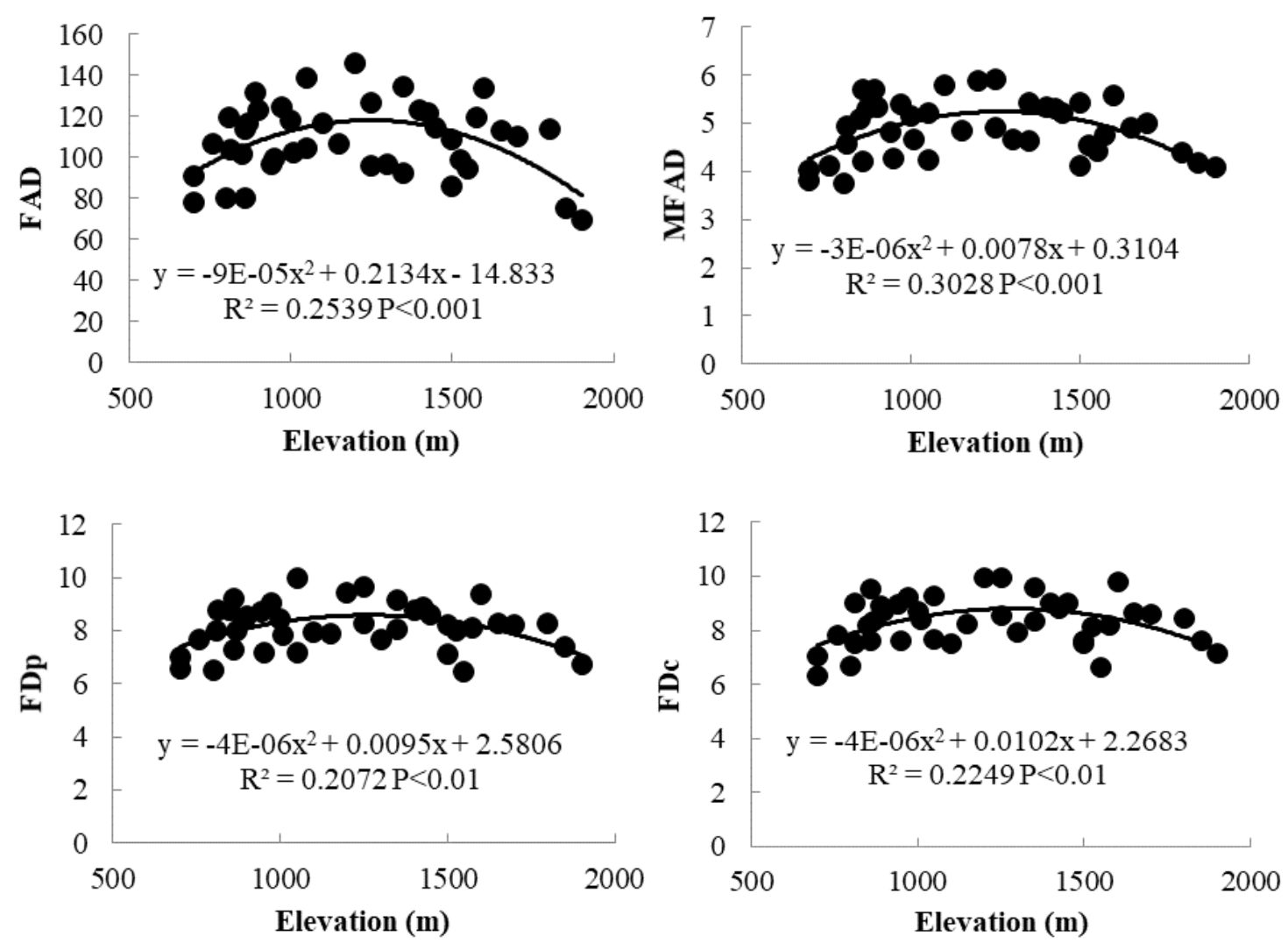

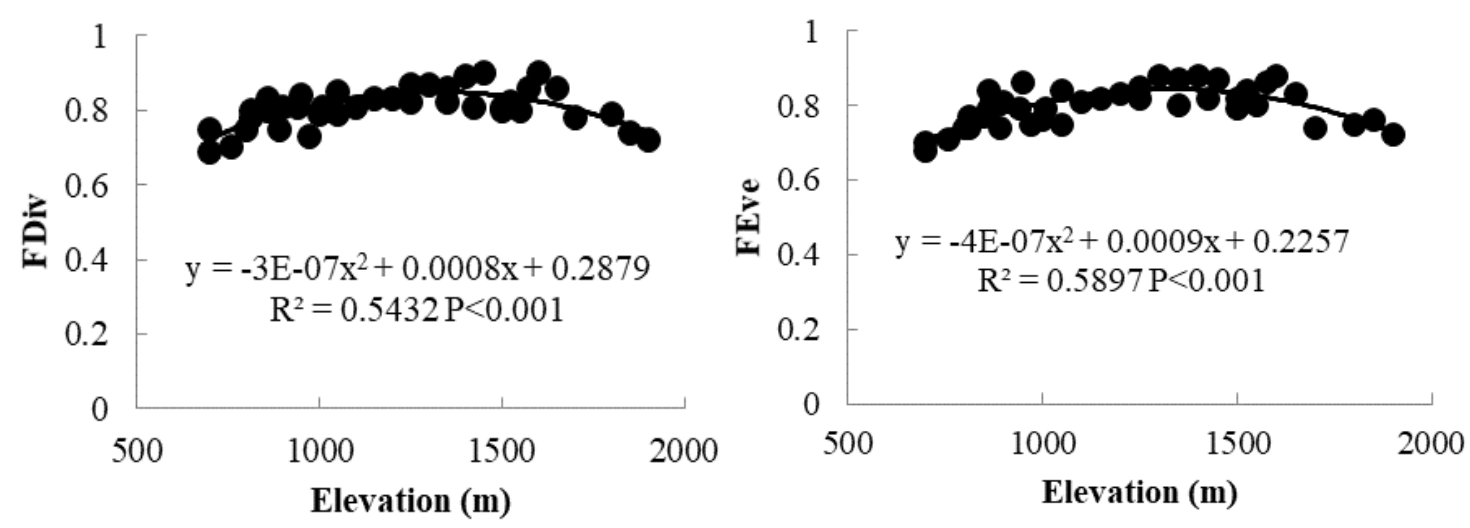

Figure 2. Variation of functional diversity in forest communities along the altitudinal gradient in the Wulingshan Nature Reserve, North China

Functional diversity indices were significantly correlated with an altitudinal gradient (Fig. 2) and with a disturbance intensity gradient (Fig. 3) in the Wulingshan Nature Reserve. All six indices showed similar variation pattern with altitudinal and disturbance gradients. Functional diversity showed a non-linear "humped" change pattern along the altitudinal gradient, i.e. it increased with elevation firstly, and reached its maximum between 1300 and $1400 \mathrm{~m}$, and then decreased gradually (Fig. 2). Functional diversity showed a non-linear or near linear decrease pattern along the disturbance intensity gradient, i.e. it was gradually decreased with the maximum value at the lowest disturbance community and the minimum value under the heaviest disturbance (Fig. 3).

In addition to altitude and disturbance, litter thickness was also significant in affecting spatial variation of functional diversity in the Wulingshan Reserve (Table 2). The litter thickness played a special role in two functional diversity indices, FEve and FDiv. However, the soil depth, slope, and aspect were not significant in influencing functional diversity change in this reserve (Table 2). Different indices showed different significance for their relationships with functional diversity, but the positive or negative feature of the relationships was clear. Elevation, soil depth and litter thickness showed positive correlations, and disturbance and aspect negative correlations with the functional diversity indices (Table 2).

Table 2. Spearman correlation coefficients between functional diversity and environmental variables in the Wulingshan Nature Reserve, North China

\begin{tabular}{c|c|c|c|c|c|c}
\hline Environmental variables & FAD & MFAD & FDp & FDc & FEve & FDiv \\
\hline Elevation & 0.053 & 0.084 & 0.077 & 0.109 & $0.374^{*}$ & $0.321^{*}$ \\
Slope & 0.232 & 0.222 & 0.133 & 0.013 & -0.226 & -0.188 \\
Aspect & -0.175 & -0.170 & -0.189 & -0.276 & 0.004 & -0.040 \\
Litter thickness & 0.199 & 0.212 & 0.178 & 0.197 & $0.440^{* *}$ & $0.414^{* * *}$ \\
Soil depth & 0.137 & 0.103 & 0.156 & 0.152 & 0.297 & 0.268 \\
Disturbance intensity & $-0.342^{*}$ & $-0.414^{* *}$ & $-0.368^{*}$ & $-0.367^{*}$ & $-0.747^{* * *}$ & $-0.739^{* * *}$ \\
\hline
\end{tabular}

Functional diversity was significantly correlated with species diversity (Table 3). The Shannon-Wiener index was most significantly correlated with all the functional 
diversity indices, species richness significantly correlated with all functional indices except FDp and FDiv, and species evenness significantly correlated with all functional indices except FDp and FD, in the Wulingshan Reserve (Table 3). Functional diversity indices were positively related to species richness, diversity and evenness, i.e. functional diversity increased with species diversity increase.
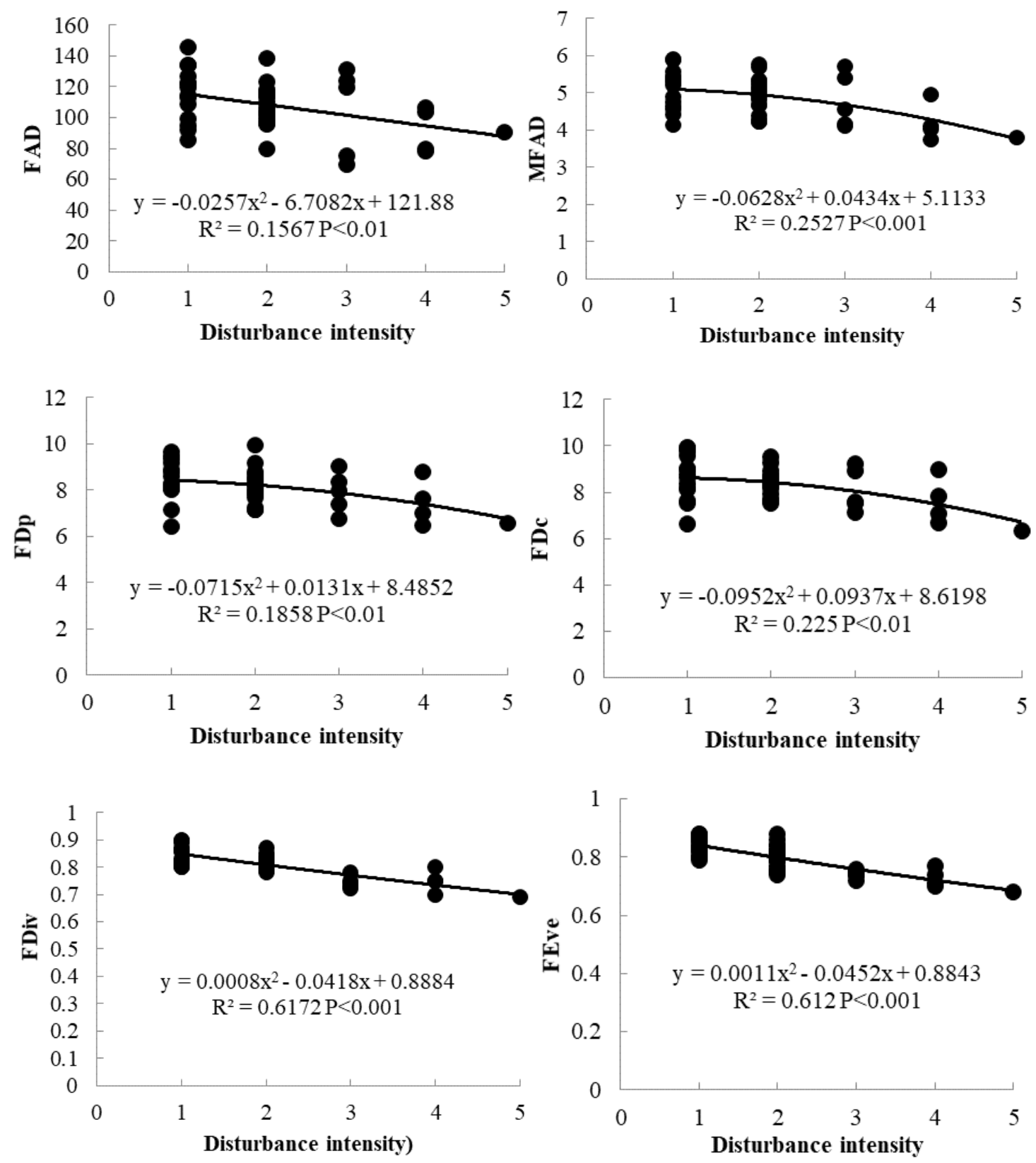

Figure 3. Change of functional diversity in forest communities along the disturbance intensity gradient in the Wulingshan Nature Reserve, North China

The six functional diversity indices were significantly correlated with each other and hence provided similar results (Table 4). Among them, MFAD, FEve and FDiv provided better correlations with elevation and disturbance. 
Table 3. Spearman correlation coefficients between functional diversity and species diversity in the Wulingshan Nature Reserve, North China

\begin{tabular}{c|c|c|c|c|c|c}
\hline Species diversity & FAD & MFAD & FDp & FDc & FEve & FDiv \\
\hline Species richness & $0.338^{*}$ & $0.374^{*}$ & 0.278 & $0.350^{*}$ & $0.330^{*}$ & 0.277 \\
Shannon-Weiner index & $0.509^{* * *}$ & $0.483^{* *}$ & $0.317^{*}$ & $0.367^{*}$ & $0.433^{* *}$ & $0.406^{* *}$ \\
Species evenness & $0.369^{*}$ & $0.339^{*}$ & 0.184 & 0.208 & $0.442^{* *}$ & $0.392^{*}$ \\
\hline
\end{tabular}

Table 4. Spearman correlation coefficients between functional diversity indices in the Wulingshan Nature Reserve, North China

\begin{tabular}{c|c|c|c|c|c|c}
\hline & FAD & MFAD & FDp & FDc & FEve & FDiv \\
\hline FAD & 1.000 & & & & & \\
MFAD & $0.815^{* * *}$ & 1.000 & & & & \\
FDp & $0.775^{* * *}$ & $0.826^{* * *}$ & 1.000 & & & \\
FDc & $0.724^{* * *}$ & $0.743^{* * *}$ & $0.921^{* * *}$ & 1.000 & & \\
FEve & $0.320^{*}$ & $0.405^{* *}$ & $0.322^{*}$ & $0.400^{* *}$ & 1.000 & \\
FDiv & $0.341^{*}$ & $0.441^{* *}$ & $0.390^{*}$ & $0.444^{* *}$ & $0.948^{* * *}$ & 1.000 \\
\hline
\end{tabular}

\section{Discussion}

Functional diversity can be used as an indicator of ecosystem functioning and can demonstrate the relationships of species function, composition, structure and environmental factors (de Bello et al., 2006; Spasojevic et al., 2014). The six functional diversity indices all showed a great change of functional diversity in forest communities in the Wulingshan Nature Reserve, which suggested that forest communities varied in species function, composition, diversity, structure and inner environment (Zhang et al., 2013; Rossi et al., 2020). Eight forest formations were identified from 700 to $1900 \mathrm{~m}$ in this reserve (Liu and Xi, 1997; Zhang et al., 2014). These vegetation formations were as follows from lower to upper hills, Form. Pinus tabulaeformis, Form. Juglans mandshurica, Form. Betula platyphylla, Form. Quercus mongolica, Form. Betula dahurica + Populus davidiana + Betula platyphylla, Form. Popolus davidiana, Form. Tilia mandschurica + Tilia mongolica. Form. Larix principis-rupprechtii (Cui et al., 2008; Zhang et al., 2014). The variation of functional diversity successfully reflected the change of forest communities along the altitudinal gradient, which suggests that each community has special functional composition and diversity (Zhang et al., 2014), which was also proved by other studies (e.g. Perronne et al., 2014; Zhang et al., 2013, 2015a).

The variation of functional diversity is usually related to changes in environmental gradients (Zhang et al., 2015b; Bricca et al., 2019). All the six indices were significantly correlated with altitudinal and disturbance gradients in the Wulingshan Nature Reserve, which suggested that elevation and disturbance were key factors influencing functional diversity in this reserve (de Bello et al., 2006; Fayiah et al., 2019). This was identical with the conclusion that altitude and disturbance were most important factors affecting forest structure, diversity and distribution in this reserve (Liu and Xi, 1997; Cui et al., 2008). All the six functional diversity indices showed the same variation pattern, nonlinear "humped" change, along the altitudinal gradient, i.e. it increased firstly, and reached its maximum between 1300 and $1400 \mathrm{~m}$, and then decreased with elevation 
increase (Zhang et al., 2013; Boehnke et al., 2014). This change pattern of functional diversity is consistent with the most commonly observed pattern that the maximum diversity to be appeared at intermediate altitude (Austrheim, 2002; Villéger et al., 2008). Altitudinal gradient represents a comprehensive gradient of heat and moisture in mountains, i.e. the change of heat and moisture with elevation will influence the change pattern of functional diversity (Zhang et al., 2013; Perronne et al., 2014). All the six indices showed the same pattern, non-linear or near linear decrease pattern, along the disturbance intensity gradient in the Wulingshan Nature Reserve. The maximum functional diversity appeared at the lowest disturbance and the minimum functional diversity was under the heaviest disturbance (Zhang et al., 2014). This pattern of functional diversity is not consistent with the hypothesis that the maximum diversity to be appeared at medium disturbance (Spasojevic et al., 2014). Disturbance from tourist activities such as trampling, damaging plant, rubbish etc. can affect functional diversity directly, and it can also influence functional diversity indirectly by changing environmental features such as soil physical characteristics (Duivenvoorden and Cuello, 2012).

Besides elevation and disturbance, soil litter thickness was significantly affecting functional diversity in the Wulingshan Nature Reserve, which was supported by some other studies (Duivenvoorden and Cuello, 2012; Zhang et al., 2014). Litter thickness affects soil water and soil nutrients which further affect functional diversity (Otypkova et al., 2011). Soil depth, slope, and aspect was not significant in influencing functional diversity change in this reserve. Former studies showed that forest vegetation was significantly correlated with slope, aspect and soil depth in this reserve (Liu and $\mathrm{Xi}$, 1997; Cui et al., 2008), which suggests that the formation and maintain of functional diversity in community is different from that of vegetation and species diversity (Zhang et al., 2013) and needs special attentions in conservation (Boehnke et al., 2014).

Functional diversity was significantly correlated with species diversity in the Wulingshan Reserve, which is consistent with many other studies (Ricotta and Moretti, 2008; de Bello et al., 2009). Functional diversity and species diversity were interrelated with each other, but they were different and could not be replaced with each other (Butterfield and Suding, 2013; Zhang et al., 2015a).

The six common indices used for measuring functional diversity were all effective in quantifying functional diversity and its variation in forest communities in the Wulingshan Nature Reserve. These indices were significantly correlated with each other since they all based on the functional distances between species in functional trait space with some theoretically differences (Casanoves et al., 2011; Song and Zhang, 2013). MFAD, FEve and FDiv performed better in describing the relationships of functional diversity with elevation and disturbance, and should be firstly used in research practice.

\section{Conclusions}

Functional diversity showed a great change in forest communities in the Wulingshan Nature Reserve, China. Elevation and disturbance were most important factors influencing functional diversity in forest communities. Functional diversity showed a non-linear "humped" change pattern along the altitudinal gradient and a non-linear or near linear decrease pattern along the disturbance intensity gradient. Functional diversity was significantly correlated with species diversity in forests in this reserve. The interactions between functional diversity, species diversity and phylogenetic 
diversity should be further studied in the forest communities. Among the six common indices used, MFAD, FEve and FDiv should be recommended in practice in future study.

Acknowledgements. This study was financially supported by the Young Teachers Found of Beijing University of Chinese Medicine (Grant No. 2018-JYB-JS) and the National Natural Science Foundation of China (No. 31170494).

\section{REFERENCES}

[1] Albert, C. H., de Bello, F., Boulangeat, I., Pellet, G., Lavorel, S., Thuiller, W. (2012): On the importance of intraspecific variability for the quantification of functional diversity. Oikos 121: 116-126.

[2] Austrheim, G. (2002): Plant diversity patterns in semi-natural grasslands along an elevational gradient in southern Norway. - Plant Ecology 161(2): 193-205.

[3] Bai, S., Lu, G., Gu, J., Zhang, S., Zheng. H., Wang. X. (2006): Studies on species and landscape pattern diversities of Wuling Mountain. - Journal of Agricultural University of Hebei (2): 1-4.

[4] Boehnke, M., Kroeber, W., Welk, E., Bruelheide. H. (2014): Maintenance of constant functional diversity during secondary succession of a subtropical forest in China. Journal of Vegetation Science 25: 897-911.

[5] Bricca, A., Conti, L., Tardella, M. F., Catorci, A., Iocchi, M., Theurillat, J. P., Cutini, M. (2019): Community assembly processes along a sub-Mediterranean elevation gradient: analyzing the interdependence of trait community weighted mean and functional diversity. - Plant Ecology 220: 1139-1151. DOI: 10.1007/s11258-019-00985-2.

[6] Butterfield, B. J., Suding, K. N. (2013): Single-trait functional indices outperform multitraitindices in linking environmental gradients and ecosystem services in a complex landscape. - Journal of Ecology 101: 9-17.

[7] Casanoves, F., Pla, L., Di Rienzo, J., Diaz, S. (2011): Diversity: a software package for the integrated analysis of functional diversity. - Methods in Ecology Evolution 2: 233237.

[8] Cui, G. F., Xing, S. H., Zhao, B. (2008): Conservation of Mountain Plants and Vegetation in Beijing. - China Forestry Press, Beijing (in Chinese).

[9] de Bello, F., Lepš, J., Sebastia, M. T. (2006): Variations in species and functional plant diversity along climatic and grazing gradients. - Ecography 29: 801-810.

[10] de Bello, F., Thuiller, W., Lepš, J., Choler, P., Clement, J., Macek, P., Sebastia, M.-T., Lavorel, S. (2009): Partitioning of functional diversity reveals the scale and extent of trait convergence and divergence. - Journal of Vegetation Sciences 20: 475-486.

[11] Duan, D. (2009): Study on the diversity of wild plants resources in Wuling Mountain and their garden application evaluation. - Journal of Anhui Agricultural Sciences (11): 8-10.

[12] Duivenvoorden, J. F., Cuello, N. L. (2012): Functional trait state diversity of Andean forests in Venezuela changes with altitude. - Journal of Vegetation Sciences 23: 11051113.

[13] Fayiah, M., Dong, S. K., Li, Y., Xu, Y. D., Gao, X. X., Li, S., Shen, H., Xiao, J. N., Yang, Y. F., Wessell, K. (2019): The relationships between plant diversity, plant cover, plant biomass and soil fertility vary with grassland type on Qinghai-Tibetan Plateau. Agriculture Ecosystems and Environment 286. DOI: 10.1016/j.agee.2019.106659.

[14] He, S. Y., Xing, Q. H., Yin, Z. T. (1992): Flora of Beijing. - Beijing Press, Beijing (in Chinese).

[15] Laliberte, E., Legendre, P. (2010): A distance-based framework for measuring functional diversity from multiple traits. - Ecology 91: 299-305. 
[16] Liu, J., Xi, W. (1997): The main vegetation types and vertical distribution laws in Wulingshan Mountains. - Journal of Capital Normal University 18(1): 95-103.

[17] Muhumuza, M., Byarugaba, D. (2009): Impact of land use on the ecology of uncultivated plant species in the Rwenzori mountain range, mid western Uganda. - African Journal of Ecology 47: 614-621.

[18] Otypkova, Z., Chytry, M., Tichy, L., Pechanec, V., Jongepier, J. W., Hajek, O. (2011): Floristic diversity patterns in the White Carpathians Biosphere Reserve, Czech Republic. - Biologia 66: 266-274.

[19] Pavoine, S., Bonsall, M. B. (2011): Measuring biodiversity to explain community assembly: a unified approach. - Biological Review 86: 792-812.

[20] Perronne, R., Mauchamp, L., Mouly, A., Gillet, F. (2014): Contrasted taxonomic, phylogenetic and functional diversity patterns in semi-natural permanent grasslands along an altitudinal gradient. - Plant Ecology and Evolution 147: 165-175.

[21] Petchey, O., Gaston, K. (2002): Functional diversity (FD), species richness and community composition. - Ecology Letters 5(3): 402-411.

[22] Petchey, O., Gaston, K. (2006): Functional diversity: back to basics and looking forward. - Ecology Letters 9(6): 741-758.

[23] Ricotta, C., Moretti, M. (2008): Quantifying functional diversity with graph-theoretical measures: advantages and pitfalls. - Community Ecology 9(1): 11-16.

[24] Rossi, C., Kneubuler, M., Schutz, M., Schaepman, M. E., Haller, R. M., Risch, A. C. (2020): From local to regional: functional diversity in differently managed alpine grassland. - Remote Sensing of Environment 236. DOI: 10.1016/j.rse.2019.111415.

[25] Song, N. Q., Zhang, J.-T. (2013): An index for measuring functional diversity in plant communities based on neural network theory. - Journal of Applied Mathematics Article ID 320905.

[26] Spasojevic, M. J., Copeland, S., Suding, K. N. (2014): Using functional diversity patterns to explore metacommunity dynamics: a framework for understanding local and regional influences on community structure. - Ecography 37: 939-949.

[27] Villéger, S., Mason, N. W. H., Mouillot, D. (2008): New multidimensional functional diversity indices for a multifaceted framework in functional ecology. - Ecology 89: 22902301.

[28] Walker, B. H., Kinzig, A., Langridge, J. L. (1999): Plant attribute diversity, resilience, and ecosystem function: the nature and significance of dominant and minor species. Ecosystems 2: 95-113.

[29] Xiang, C. L., Zhang, J.-T. (2009): Changes in species diversity and contributing factors in subalpine meadows in Dongling Mountain. - Journal of Beijing Normal University (Natural Science) 45(3): 275-278.

[30] Zhang, J.-T., Xu, B., Li, M. (2013): Vegetation patterns and species diversity along elevational and disturbance gradients in the Baihua Mountain Reserve, Beijing, China. Mountain Research and Development 33(2): 170-178.

[31] Zhang, J.-T., Li, M., Nie, E. B. (2014): Pattern of functional diversity along an altitudinal gradient in the Baihua Mountain Reserve of Beijing, China. - Brazilian Journal of Botany 37: 37-45.

[32] Zhang, J.-T., Xiao, J., Li, L. F. (2015a): Variation of plant functional diversity along a disturbance gradient in mountain meadows of the Donglingshan Reserve, Beijing, China. - Russian Journal of Ecology 46(2): 157-166.

[33] Zhang, J.-T., Zhang, B., Qian, Z. Y. (2015b): Functional diversity of Cercidiphyllum japonicum communities in the Shennongjia Reserve, central China. - Journal of Forestry Research 26(1): 171-177.

[34] Zhang, W. M., Qin, A. C., Feng, X. Q., Li, D. Y., Huang, D. Z. (2003): Evaluation of tourist resources in Wulingshan Reserve. - Hebei Research of Forest and Fruit 18(2): 184-189 (in Chinese). 